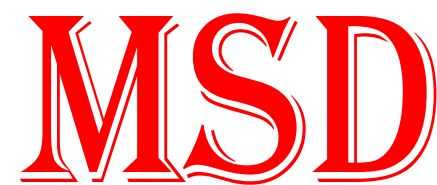

Medical Science and Discovery ISSN: 2148-6832

\title{
Acute and chronic toxicity of ethyl chloride insufflation in two patients
}

\author{
Jericha Viduya ${ }^{1}$, Jeffrey M Levine ${ }^{2 *}$ \\ 1 Dept of Psychiatry, University of California Irvine School of Medicine, Irvine, CA, USA \\ 2 Dept of Psychiatry and Neurosciences, University of California Riverside School of Medicine Riverside, \\ CA, USA \\ * Corresponding Author: Jeffrey M Levine E-mail: drjlevinewestport@ gmail.com
}

\section{ABSTRACT}

Objective: Inhalant abuse has been a source of increasing concern because of its easy accessibility and affordability. Anecdotal reports have previously described ethyl chloride as a potential cause of altered mental state and neurologic symptoms. Its use has been thought to be found most often in adolescents and among men who have sex with men. Common acute symptoms include confusion, dizziness, headache, nausea, and fatigue. We describe two cases of adult patients who presented to one general hospital emergency department with ethyl chloride toxicity. The first presented with acute delirium; the second with a picture of chronic neurological symptomatology. It is important that clinicians become familiar with ethyl chloride intoxication because of its prevalence and potential to present with varying acute and chronic symptomatology.

Key words: Inhalation exposure, ethyl chloride, drug toxicity

\section{INTRODUCTION}

Inhalant abuse has been an increasing source of concern because of its easy accessibility and affordability (1). Ethyl chloride is a clear, colorless volatile vapour once used as a general anesthetic but which is now commonly found and readily available in spray form as a topical anesthetic, refrigerant, and solvent. Its use as a recreational inhalant is believed to be increasing although its precise prevalence is unknown. Most reports focus on the abuse of ethyl chloride as a euphoriant among adolescents and young adults $(2,3)$. Documented symptoms of acute ethyl chloride inhalation include confusion, dizziness, headache, nausea, and fatigue. Long-term insufflation of ethyl chloride can cause significant neurotoxicity including visual hallucinations, nystagmus, dysarthria, lack of voluntary muscle control, intention tremors, and ataxia. We here describe two middleaged adults who presented at one institution and who illustrate the effects of both acute and chronic abuse.

\section{Case of acute ethyl chloride inhalation}

A 49-year-old man with a history of bipolar disorder was brought to the emergency department with drowsiness, agitation, and confusion. According to the patient's family, he was in his usual state of health until one week prior this presentation at which time he developed slurred speech and emanated a strange odor. He exhibited increasing confusion over the next several days including evident visual hallucinations and the delusion that he had been evicted from his home. He was noted to be packing his belongings to prepare for this imagined eventuality.

On the day of admission, patient's family found a total of 12 empty cans of ethyl chloride in the garbage bin next to the patient's bed. The patient was noted to have a blank stare with drooping eyelids, unintelligible speech, and rapid breathing. He was transferred to the emergency department. On physical examination, the patient was awake but agitated. Vital signs were notable for tachycardia of $110 \mathrm{bpm}$ and tachypnea with a respiratory rate of 30. He was afebrile and normotensive. Routine urine toxicology screening was negative. Serum concentration of ethyl alcohol was less than $10 \mathrm{mg} / \mathrm{dL}$. Chemistry and arterial blood gas determinations revealed a mixed acid-base disturbance with severe respiratory alkalosis and metabolic acidosis with a $\mathrm{pH}$ of $7.38, \mathrm{pO}_{2}$ of 71 , and $\mathrm{pCO}_{2}$ of 16 but with a normal anion gap. Electrocardiography displayed a normal sinus rhythm. Neuroimaging was not performed. 
Shortly after admission, patient began to have increased secretions, compromising his airway. He required endotracheal intubation and was transferred to the intensive care unit (ICU). During the ensuing 48 hours, his alertness improved, and his agitation abated. He was oriented and spoke in complete sentences.

His clinical course in the ICU was complicated only by mild azotemia that resolved over the next several days. He was placed back on his baseline psychiatric medications of bupropion and quetiapine. He was discharged in a stable and improved state. Two weeks after discharge, the patient was seen at an outpatient clinic. He reported abstinence from ethyl chloride inhalation and was observed to have resolution of his psychiatric and respiratory symptoms.

\section{Case of chronic ethyl chloride inhalation}

A 62-year-old man with a history of major depression and anxiety presented to the emergency department with newonset diplopia of one-month duration. He also reported upper extremity tremors, tongue spasticity, and gait instability. One month prior to his presentation, the patient was seen at an outpatient clinic and was noted to have ataxia and memory impairment, which continued to persist. The patient's past medical history was significant for alcohol and substance dependency, with a self-reported abstinence of more than a decade.

On physical examination, the patient was awake and alert. Vital signs were within normal limits. The patient's thinking was tangential, and he was observed at times to respond to internal stimuli. On cognitive assessment, the patient was oriented to person but not to place or time. Memory and clock drawing were markedly impaired.

The patient had full extraocular movements without nystagmus but right-sided facial droop with nasolabial fold flattening, slurred speech, intention tremor, wide-based ataxia, diffuse hyperreflexia and a positive Romberg sign. Laboratory results were unremarkable. Urine drug screen was positive for cannabinoids only. Serum ethanol level was undetectable. Electrocardiogram incidentally revealed the presence of atrial flutter with rapid ventricular response. A subsequent nuclear stress test was negative. Both non-contrast CT of the head and MRI of the brain were negative.

After finding no clear aetiology for his symptoms, a more detailed history was pursued by the medical resident. The patient revealed the chronic use of ethyl chloride several times per week for one year. The patient received supportive therapy for his symptoms.

He was started on an antipsychotic, olanzapine $5 \mathrm{mg}$ by mouth daily, for his disorganization. His atrial flutter returned to normal sinus rhythm after cardioversion and amiodarone therapy. Over the following several days, the patient's upper extremity tremors, tongue spasticity, and gait instability resolved.

The patient was advised to discontinue ethyl chloride use. Olanzapine was discontinued. The patient has been followed by his primary care physician and psychiatrist for several months without any recurrence of neurological symptoms.

\section{DISCUSSION}

Ethyl chloride $\left(\mathrm{C}_{2} \mathrm{H}_{5} \mathrm{Cl}\right)$, or chloroethane, is a colorless volatile vapor with a characteristic ether-like odor.4 It has historically been used as a general anesthetic in major surgeries. Due to the risk of accidental death, ethyl chloride is no longer medically used to induce a state of sedation. Currently, it is used as a topical anesthetic in the form of a spray. It is also used as a refrigerant, solvent, and alkylating agent.

Ethyl chloride can be purchased both online and in stores as a legitimate household product or as drug paraphernalia (e.g., Maximum Impact, Black Max Heavy Duty, Black Jac, Ethyl Gaz, Ethyl Four Star, Jungle Juice Plus, Rush, and Macho). Hence, it has increasingly become an agent of choice for recreational "sniffing" $(5,6)$. A Singapore American School newspaper released a video report that demonstrated the ease of obtaining "EC" by high school students due to its easy accessibility and affordability. A student reported that when he got high from "EC", he felt as if everything slowed down, that he became more aware of his heartbeat, and that he simply could not keep himself from laughing (7) Inhalants are the second most prevalent illicit substance among 8th graders in the United States according to the Monitoring the Future study in 2018 by the University of Michigan (2, 3). Although the prevalence of ethyl chloride use among adults is not well documented, there are reports of its use and abuse to enhance sexual pleasure among men who have sex with men (8).

Inhaled ethyl chloride is readily absorbed through the lungs. Its lipophilic nature allows it to cross the blood brain barrier, accumulating in the brain at a concentration two times that of blood (9) Studies on rats have shown that ethyl chloride is metabolized by cytochrome P450 to acetaldehyde and is then metabolized either to acetic acid or, by the conjugation of ethyl chloride with glutathione, to S-ethyl-glutathione, which is excreted in the urine.

Unmetabolized ethyl chloride is exhaled through the lungs accounting for its characteristic odor. Animal studies have also shown that ethyl chloride can enhance the effects of alcohol (5). This finding is consistent with the observation that at acute high levels of ethyl chloride gas exposure, temporary feelings of drunkenness, lack of muscle coordination, and unconsciousness are produced. Other potential symptoms of acute overexposure include cardiac arrhythmias and cardiac arrest $(4,5)$.

Long-term insufflation of ethyl chloride can also cause significant neurotoxicity. Symptoms that have been reported related to chronic abuse include visual hallucinations, nystagmus, dysarthria, lack of voluntary muscle control, intention tremors, and ataxia $(\mathbf{6 , 1 0 - 1 3 )}$. There are 3 reported deaths due to ethyl chloride insufflation $(1,14,15)$.

Our first patient's non-anion gap metabolic acidosis was likely due to ethyl chloride toxicity, similar to the non-anion gap metabolic acidosis reported with toluene inhalation. In toluene metabolism and elimination, the metabolite hippurate is filtered and secreted into the renal tubule and then rapidly excreted in the urine, preventing a plasma build-up of organic acids, which would lead to an increased anion gap (17). 
This explanation is further supported as the acid-base disorder rapidly resolved after ethyl chloride abstinence.

Studies in dogs have shown cardiac irregularities from acute exposure to anaesthetic concentrations of ethyl chloride (15, 16). Given that the second patient had no significant cardiac history or other identifiable aetiology, his atrial flutter was most likely associated with ethyl chloride inhalation. While cannabis may cause mental status changes, intoxication is often short-lived and does not produce the neurological signs and symptoms seen in this patient.

Diagnosis is based on high clinical suspicion and unremarkable imaging and laboratory workup (except, perhaps, the above noted non-anion gap acidosis). There are no commonly used medical tests available to determine exposure to ethyl chloride. One recent forensic study presented a 40-year-old man found unresponsive after ethyl chloride inhalation, confirmed by performing a dynamic headspace gas chromatography coupled to mass spectrometry. Ethyl chloride levels were detected in peripheral and central blood and lung and brain tissues (1). However, while gas chromatography exists and can be used for confirmation by measuring ethyl chloride levels in blood, milk, and urine, it is not readily available in the clinical setting.

The mainstay of managing ethyl chloride intoxication has been largely based on supportive care measures. In reported cases of chronic inhalation of ethyl chloride that resulted in neurotoxicity, symptoms resolved days to weeks after cessation of ethyl chloride inhalant abuse, similar to the course our second patient. It has been noted that unconscious patients breathing spontaneously may recover by the time they arrive in the emergency department if the toxic state is due to isolated ethyl chloride toxicity. Persistent unconsciousness should prompt assessment of the presence of other substances of abuse. There appears to be no withdrawal state associated with the discontinuation of ethyl chloride use.

\section{CONCLUSION}

We believe that both our patients' neurotoxic symptoms were associated with ethyl chloride insufflation. Both had unremarkable urine drug screens. Both patients admitted to using ethyl chloride, and cessation resulted in the resolution of symptoms, as noted in other published case reports.

Physicians should become familiar with the toxic symptoms associated with ethyl chloride and other inhalants of abuse. Acute presentations can manifest as delirium, acid-base abnormalities, hyperventilation, or cardiac arrhythmias. More chronic use appears to display more focal neurologic signs such as tremors, ataxia, and hyperreflexia. Atrial or ventricular arrhythmias, including reports of ventricular fibrillation, are possible with either acute or chronic use. Although inhalant abuse has been shown to be most prevalent among adolescents, clinicians should recognize a growing use among older patients as well, particularly among men who have sex with men. Supportive measures are the mainstay of management. Efforts directed towards rehabilitation, psychiatric evaluation, and counselling for substance abuse are of key importance. Indeed, since many cases demonstrate a near-complete recovery following discontinuation of ethyl chloride insufflation, stressing cessation can prevent the very serious consequences of ethyl chloride abuse.
Author Contributions: JV, JML: Research of the literature, Patient examinations, Manuscript preparation and revisions.

Financial \& competing interest's disclosure: The authors have no relevant affiliations or financial involvement with any organisation or entity with a financial interest in or financial conflict with the subject matter or materials discussed in the manuscript. This includes employment, consultancies, honoraria, stock ownership or options, expert testimony, grants or patents received or pending, or royalties.

Conflict of interest: The author declared no potential conflicts of interest with respect to the research, authorship, and/or publication of this article. This research did not receive and specific grant from funding agencies in the public, commercial, or not-for-profit sectors.

\section{REFERENCES}

1. Pascali J, Fais P, Viel G, Cecchetto G, Montisci M. Is old stuff back? A fatal case of ethyl chloride sniffing. Egyptian Journal Of Forensic Sciences. 2019; 9(1):29. https://doi.org/10.1186/s41935-019-0136-4.

2. Miech RA, Johnston LD, O'Malley PM, Bachman JG, Schulenberg JE, Patrick ME. Monitoring the future national survey results on drug use, 1975-2018: volume I, secondary school students. Ann Arbor: University of Michigan, Institute for Social Research; 2019. 586 p.

3. Schulenberg JE, Johnston LD, O’Malley PM, Bachman JG, Miech RA, Patrick ME. Monitoring the future national survey results on drug use, 1975-2018: volume II, college students and adults ages 19-60. Ann Arbor: University of Michigan, Institute for Social Research; 2019. 505 .

4. Merck, Sharpe, and Dohme. The Merck Index. An Encyclopedia of Chemicals, Drugs, and Biologicals. 13th ed. Whitehouse Station, NJ: Merck and Co., Inc.; 2001. 2165p.

5. Agency for Toxic Substances and Disease Registry (ATSDR) Toxicological profile for chloroethane (Update). Atlanta, GA: Public Health Service, U.S. Department of Health and Human Service; 1998. $20 \mathrm{p}$

6. Finch C, Lobo B. Acute inhalant-induced neurotoxicity with delayed recovery. Ann Pharmacother. 2005;39:169-172.

7. The Eye. Ethyl Chloride, or EC, is new off-the-shelf high. 2014, May 19. https://youtu.be/11Z9RKDOzOk.

8. Hall TM, Shoptaw S, Reback CJ. Sometimes poppers are not poppers Huffing as an emergen health concern among MSM substance abusers. J of Gay Lesbian Ment Health. 2014; 19:118-121.

9. Konietzko H. Chlorinated ethanes: Sources, distribution, environmental impact and health effects. Hazard Assessment of Chemicals.1984; 3:401-448.

10. Soult T, Walker J. Ethyl chloride intoxication. Am J Emerg Med 1993;11:313-315.

11. Nordin C, Rosenquist M, Hollstedt C. Sniffing of ethyl chloride - an uncommon form of abuse with serious mental and neurological symptoms. Int J Addict. 1988;23:623-627.

12. Demarest C, Torgovnick J, Sethi N, Arsura E, Sethi P. Acute reversible neurotoxicity associated with inhalation of ethyl chloride: a case report. Clin Neurol Neurosurg. 2011;113:909-910.

13. Broussard L, Broussard A, Pittman T, Lirette D. Death due to inhalation of ethyl chloride. J Forensic Sci. 2000;45:223-225.

14. Yacoub I, Robinson C, Simmons G, Hall M. Death attributed to ethyl chloride. J Anal Toxicol. 1993;17:384-385. 
15. Haid B, White JM, Morris LE. Observations of cardiac rhythm during ethyl chloride anesthesia in the dog. Curr Res Anesth Analg. 1954; 33(5):318-325.

16. Morris TE, Tasto WD. 1979. Ethyl chloride. In: Grayson M, Eckroth D, eds. Kirk-Othmer encyclopedia of chemical technology. 3rd ed. Vol. 5. New York, NY: John Wiley and Sons. p714-722.
17. Carlisle EJ, Donnelly SM, Vasuvattakul S, Kamel KS, Tobe S, Halperin ML. Glue-sniffing and distal renal tubular acidosis: sticking to the facts. J Am Soc Nephrol 1991; 1(8):1019-1027.

Copyright (C) 2021 The Author(s); This is an open-access article distributed under the terms of the Creative Commons Attribution License (http://creativecommons.org/licenses/by/4.0), (CC BY NC) which permits unrestricted use, distribution, and reproduction in any medium, provided the original work is properly cited. International Journal of Medical Science and Discovery. 